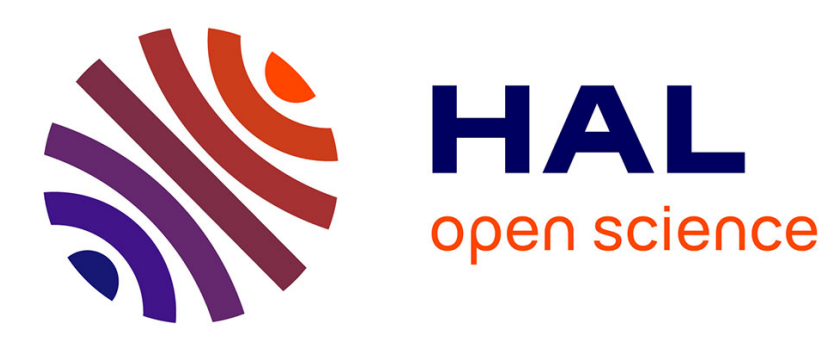

\title{
Sur la théorie des perturbations de Rayleigh
}

J. Winter

\section{To cite this version:}

J. Winter. Sur la théorie des perturbations de Rayleigh. J. Phys. Radium, 1935, 6 (12), pp.516-520. 10.1051/jphysrad:01935006012051600 . jpa-00233374

\section{HAL Id: jpa-00233374 https://hal.science/jpa-00233374}

Submitted on 1 Jan 1935

HAL is a multi-disciplinary open access archive for the deposit and dissemination of scientific research documents, whether they are published or not. The documents may come from teaching and research institutions in France or abroad, or from public or private research centers.
L'archive ouverte pluridisciplinaire HAL, est destinée au dépôt et à la diffusion de documents scientifiques de niveau recherche, publiés ou non, émanant des établissements d'enseignement et de recherche français ou étrangers, des laboratoires publics ou privés. 


\title{
SUR LA THÉORIE DES PERTURBATIONS DE RAYLEIGH
}

\author{
Par J. WINTER.
}

\begin{abstract}
Sommaire. - Nous supposons connue du lecleur la théorie des perturbations de Rayleigh qui, le problème spectral (détermination des fonctions propres et des valeurs propres) étant résolu pour un Hamiltonien $H^{0}$, cherche à le résoudre pour un Hamiltonien voisın $H^{\prime}+H\left({ }^{1}\right)$. Nous voulons étudier les propriétés de convergence des diverses approximations. 11 est bien entendu nécessaire pour cela. de donnel au terme $H^{j}$ des formes particulières, sur lesquelles les résultats sont simples à démontrer. Lorsque nous chercherons à passer aux applications physiques, $H^{0}$ et $H^{1}$ seront imposés et nous sortons des cas simples de l'étude théorique. Il est alors nécessaire d'abandonner la rigueur mathématique et de procéder d'une m ınière intuitive. Pais, n jus rovenons, encore une fois, aux phénomènes de diffusion, espérant éclairer la móthods de porturbation de Born, en la rapprochant de celle de Rayleigh.
\end{abstract}

1. Problème simplifié. - A. Formules générales. - Nous adoptons la notation des matrices et suivons, ici, l'exposé du livre déjà cité de Born et Jordan : Nous partons d une matrice. $\boldsymbol{H}^{0}(p, q)$, ayant un nombre fini de lignes et de colonnes, $p$ et $q$ sont des matrices canoniques $\left({ }^{2}\right)$ et nous supposons que $\boldsymbol{H}^{n}$ est diagonale.

$$
H^{0}(p, q)=W^{0} \text {. }
$$

Il s'agit de trouver une transformation unitaire $U$, rendant diagonale la matrice $H^{0}+H^{\prime}(p, q)$, où $\boldsymbol{H}^{\prime}$ représente la perturbation, et de calculer les nouvelles valeurs spectrales. Pour cela, on suppose $\boldsymbol{H}^{1}$ multiplié par un coefficient arbitraire $\curlywedge$, et on écrit $\left({ }^{3}\right)$

$$
\left.\begin{array}{c}
U=1+\lambda U^{1}+\lambda^{2} U^{2}+\ldots . \\
W=W^{0}+\lambda W^{1}+\lambda W^{2}+\ldots
\end{array}\right\}
$$

On obtient, en première approximation, les formules :

$$
\begin{aligned}
& W_{n}^{1}=H_{n n}^{1} \\
& U_{n m}^{1}=-\frac{1}{W^{0}} \\
& U_{n n}^{1}=0
\end{aligned}
$$

Ces formules, et les formules générales en $k^{\grave{i} m o}$ approximation, résolvent formellement le problème.

(1) Voir : L. de Broglae. "La théorie de la quantificalıon dans la nouvelle mécanique "Hermann, 1932. - Sommerfeld. "IVellenmechanischer Ergänzungsband », p. 170 et suiv. - Braunschweig, 1930. - BonN-Jordax. "Elementare Quantenmechanik», p. 194 et suiv. Springer, 1930. - L. Brillovin a Les méthodes d'approximation en mécanique ondulatoire ", Hermann, 1932, où des problèmes analogues sont traités. - G. Julia. Cours professé à la Sorbonne 193 ว.

$\left(^{2}\right) p$ et $q$ représentent les impulsions et les coordonnées, elles vérifient les relations

$$
p_{\iota} q_{j}-q_{j} p_{\imath}=\delta_{\imath j}
$$

$$
\begin{gathered}
W_{n}^{k}=-\sum_{l=1}^{l-l}\left[U^{k-l} W^{l}\right]_{n n}+\left[H^{1} U^{k-l}\right]_{n n} \\
U_{n m}^{k}=\frac{1}{W_{n}^{0}-W_{m}^{0}}\left[\sum_{l=1}^{k-1 k-l} U^{l} W^{l}-H^{1} U^{k-1}\right] \\
U_{n n}^{k}=-\frac{1}{2} \sum_{l=1}^{k-1}\left(U^{l}+U^{k-l}\right)_{n n}
\end{gathered}
$$

Nous les appellerons formules R. (Rayleigh).

B. Hypothèses sur la matrice $H^{1}$. Appelons $\boldsymbol{E}^{\prime}$ l'espace défini par les $\boldsymbol{V}$ vecteurs propres de $\boldsymbol{H}^{0}$ et supposons que $\boldsymbol{E}$ soit la réunion de deux sous-espaces orthogonaux $E^{\prime}$ et $\boldsymbol{E}^{\prime}, \boldsymbol{E}^{\prime \prime}$ sous tendu par les vecteurs propres d'indices 1 à $P-1, E^{\prime}$ par ceux d'indices $P$ à $\Lambda$. Supposons la perturbation $H^{1}$ grande $\left(^{\prime}\right)$, mais pratiquement contenue dans $E^{\prime}$; nous voulons dire par là, que les éléments de matrice de $\boldsymbol{H}^{1}$, dans le système de coordonnées des vecteurs propres de $H^{0}$, ne sont grands que si leurs deux indices sont $\supseteq \boldsymbol{P}$; les autres seront beaucoup plus petits que ceux de $\boldsymbol{H}^{0}$. Que peut-on dire de $W_{0}^{1}$ et des composants du vecteur $l_{.0}^{1}$, qui sont $U_{0,1}^{1}$, $U_{10}^{1}, U_{20}^{1}, \ldots$ ?

Physiquement, nous voyons que ce cas se rapprochera de celui d'un champ perturbateur intense, inais s'exerçant dans une région périphérique où la fonction

$i$ el $j$ indices représentant les axes de coordonnées, is symbole de Weierstrass. Nous ne pouvons ici, reprendre la suite des raisonnements qui conduisent aux formules (1), (3), (4).

(3) Yous simplifions les notations de Born en écrivant immédiatement $p$ et $q$, au lieu de $p_{0}$ et $q_{0}$ et en supprimant les termes supérieurs du développement de $H$ qui sont inutiles, la perturbalion élanı toujours donnée exactement. On suppose que $H^{0}$ n'est pas dégénérée.

(i) Voir, à propos de grandes perturbations, L. Bricloure. " $L a$ méthode du champ self conststent ", Hermann, 1933. 
propre étudiée est de très faible amplitude. Tous les éléments de matrice où interviendront une fonction propre de petit indice, localisée près de l'origine, seront très petits. Naturellement, il n'est pas évident à priori, que dans un problème, il existe des éléments de matrice $H^{\prime}$ à deux grands indices, et Gui soient d'un ordre de grandeur supérieur à tous ceux où l'un des indices est petit. Cette hypothèse devra être vérifiée dans les applications.

Reprenons le raisonnement qui a conduit aux formules (3) mais sans faire intervenir le coefficient $\lambda . \mathrm{Au}$ lieu de $(2)$ nous écrivons :

$$
\left.\begin{array}{c}
l=1+U^{1} \\
W=W^{0}+W^{\prime}
\end{array}\right\}
$$

$W^{0}$ et $W^{1}$ sont diagonales.

On obtiendra, par un raisonnement identique à celui de Born et Jordan, au lieu des formules (3), les formules $(6)$ ( + désigne la matrice adjointe).

$$
\begin{aligned}
& W_{0}^{1}=H_{00}^{1}+\sum_{p} H_{0 p}^{1} U_{p_{0}}^{1}-U_{00}^{1} W_{0}^{1} \\
& U_{00}^{1}+U_{00}^{1+}+\sum_{p} U_{0 p}^{1} U_{p_{0}}^{1+}=0
\end{aligned}
$$$$
\text { (3) }\left(-W_{0}^{0}-W_{0}^{1}+W_{p}^{0}\right) U_{l 0}^{1}+\sum_{l} H_{p l}^{1} U_{0}^{1}=-H_{p 0}^{1}
$$

Sur l'équation $(6,3)$ mous lisons que les $U_{\mu^{0}}^{1}$ sauf $U_{00}^{1}$ sont racines d'un système d'équations qui a pour déterminant $W^{0}+H^{1}-W_{0}^{0} .1-W_{0}^{1} .1$, matrice dont on supprimera la première ligne et la première colonne. Supposons ce déterminant différent de o (1). Cela signifie que $W_{0}^{(0}+W_{0}^{1}$ ne sera pas valeur propre double (on a supposé que $W_{0}^{0}$ n'était pas racine double, mais la perturbation étant grande, la valeur propre perturbée pourrait le devenir : ce serait le cas si on perturbait le champ d'un atome à un électron de valence, par un noyau identique mais éloigné).

Si $p \leq P$. - Tous les $U_{p 0}^{1}$ seront alors infiniment petits du premier ordre comme les $\boldsymbol{H}_{p 0}{ }^{1}, U_{00}^{1}$ sera infiniment petit du $2^{e}$ ordre, et on retrouvera les formu les (3) pour $W_{0}^{1}$, et les $U_{10}^{1}$.

Pour $p \supseteq P$. - Les équations (6) ne se réduiront plus aux formu'es (3) pour les $U_{p 0}^{1}$. En effet, les coefficients $H_{p l}^{\mathrm{1}}$ ne sont plus petits pour $p$ et $/ \supseteq P$. On ne peut plus déterminer les composantes de $U_{.0}^{1}$ dinns $E$ ni aucune des valeurs propres de $E^{\prime}$.

Nous aboutissons, alors, au résultat suivant, qui est presque intuitif : Si l'on introduit une matrice de perlurbation qui n'est grande que dans un certain sous es-

(1) Nous sommes assurés à priori que $W_{0}^{1}$ existe, ce qui nous permet de raisonner ainsi. Pour ne pas allongrer, nous écartons le cas exceptionnel. pace $E^{\prime}$ et petite all dehor's, on aura, à l'aide des formules de R. les parties principales des variations des valeurs spectrales du sous-espace complementaire $E^{\prime \prime}$, orthogo. nal au premier, ainsique celles des composantes dans $\boldsymbol{E}^{\prime \prime}$ des variations des vecleurs propres correspondants. C'est tout ce qu'on peut attendre des formules de R. dans ce cas. Elles ne donnent qu'une seule approximation, et se limitent au sous-espace $E^{\prime \prime}$. On ne peut calculer de seconde' approximation $W_{0}^{2}$. On ne peut qu'en indiquer une limite supérieure. Pour aller plus loin, il faut savoir ce que se passe dans $E^{\prime}$ et les formules de R. sont incapables de nous le dire: Il faut comme point de départ supposer résolu un autre problème spectral.

2. Cas où on suppose résolu le problème spectral pour la perturbation. - La matrice $\boldsymbol{H}^{0}$ est une somme de deux termes: $T$, énergic cinélique, $S$ énergie potentielle, $\boldsymbol{H}^{4}$ représente un potentiel $\boldsymbol{V}$. Nous supposons : $1^{\circ}$ que nous sachions résoudre le problème spectral pour la matrice $T+V ; 2^{\circ}$ que l'espace total puisse être décomposé en deux sous-espaces orthogonaux $E$ et $E^{\prime \prime}$, comme plus haut, tels que les éléments de matrice de $V$ ne soient grands que dans $E^{\prime}$, ceux de $S$ dans $E^{\prime \prime}$, $E^{\prime}$ et $E^{\prime \prime}$ pouvant être indifléremment sous tendus par des vecteurs propres de $T+S$ ou de $T+V$.

Dans la première équation (i), qui donne $W_{0}^{1}$ nous. pouvons négliger le dernier terme, qui est du $3^{\mathrm{c}}$ ordre. Cherchons à évaluer le terme du $2^{\mathrm{e}}$ ordre $\sum_{p} V_{0 p} U_{p 0}^{1}$ Pour $p<\boldsymbol{P}\left(\boldsymbol{E}^{\prime}\right)$, nous pouvons appliquer la formule (4)

$$
\sum_{p<P} V_{0 p} U_{p 0}^{1}=-\sum_{p<P} \frac{V_{0 p} V_{p_{0}}}{W_{p}^{0}-W_{0}^{0}}
$$

Reprenons les équations $(6,3)$ que nous écrivons sous forme vectorielle :

$$
\left[(T+S+V) U^{1}\right]_{._{0}}-\left(\stackrel{0}{W_{0}}+W_{1}^{0}\right)_{.0}^{1}=-V_{.0}
$$

La somme $\sum_{p \supseteq P} V_{0 p} U_{p 0}^{1}=\sum_{p \leqq P} V_{p 0}^{*} U_{p^{0}}^{1}$ n'est autre, comme $V$ est évidemment hermitien, que le produit scalaire des composantes dans l'espace $\boldsymbol{E}^{\prime}$, des vecteurs $V .0$ et $U_{.0}^{1}$. Prenons pour axes dans $E^{\prime}$, les vecteurs propres de $T+V$, ce que nous savons faire, par hypothèse.

Nous désignerons les nouvelles composantes par l'indice $p^{\prime} . Z_{p^{\prime}}$ seront les valeurs propres de $T+V$. (6) deviendra, en négligeant les termes correctifs $S$ et $W_{0}^{1}$.

$$
Z_{p^{\prime}} U_{p^{\prime} 0}^{1}-W_{0}^{0} U_{p^{\prime} 0}^{1}=-V_{p^{\prime} 0}
$$

La deuxième partie de la correction sur l'énergie, ̀̀ ajouter à (7), s'écrira de mème

$$
\sum_{p \supseteq P} V_{0^{\prime}} L^{\prime \prime}{ }_{p 0}=\sum_{p^{\prime}} V_{v r^{\prime}} U_{\mu^{\prime} 0}^{1}=-\sum \frac{V_{0 p^{\prime}} V_{p^{\prime} 0}}{Z_{p^{\prime}}-W_{0}^{0}}
$$


expression que nous savons calculer par hypothèse, connaissant les vecteurs propres, et les valeurs propres $l^{\prime}$. Nous voyons que nous pouvons alors calculer complètement la correction du deuxième ordre sur les valeurs propres de l'énergie.

3. Application à des problèmes physiques simples. - a) Génèralitès. - Supposons rangées par ordre de grandeur croissante, les valeurs propres d'un problème de Schrödinger à un électron (champ Coulombien d'un noyau). Nous supposons essenliellement le domaine $D$ de l'onde fini, ce qui supprime le spectre continu. Nous obtiendrons, en portant en abscisses les indices des valeurs propres $p$, et en ordonnées leurs valeurs $W_{p}$, une suite de points ayant l'allure de la figure $1(\ldots)$.

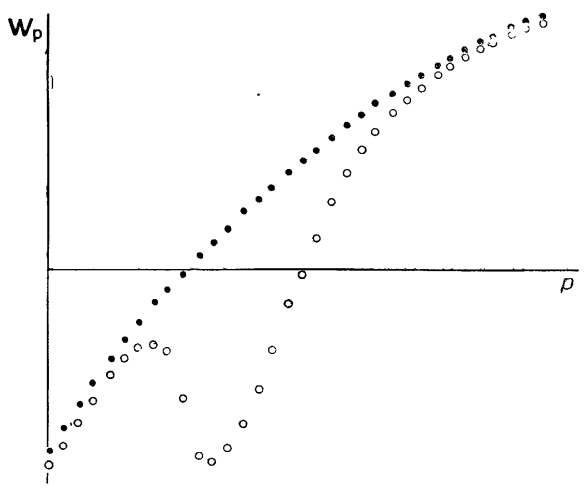

Fig. 1.

Lorsque $p$ devient infini, $H_{p}$ obéit à une loi asymptotique bien connue qui donne l'allure de la courhe $W_{p}$ et qui ne dépend plus du potentiel électrostatique du noyau $\left({ }^{1}\right)$. Introduisons un deuxième champ, d'intensité comparable au premier, par exemple celui d'un deuxième noyau, et supposé éloigné du premier. Les $\boldsymbol{I}^{r}$ de très petits indices ne seront que peu modifiés puisque le champ est éloigné. Ceux d'indices très gıands ne seront encore que peu modifiés en vertu de la loi asymptotique. Mais nous sommes certains qu'il existera des valeurs spectrales d'indices intermédiaires qui seront fortement modifiées, puisque après introduction du deuxième noyau on est sûr de retrouver les valeurs propres de petits indices, de ce noyau seul, à peine modifiées. La courbe définitive des W aura l'allure de la courbe (oooo). La figure 1 n'a qu'une valeur schématique. L'allure de la courbe perturbée n'est pas nécessairement régulière.

Si le champ perturbateur est beaucoup plus faible que le champ primitif, toutes les valeurs propres seront peu perturbées et les formules de $\mathrm{R}$ s'appliqueront, mais s'il s'agit du champ d un deuxième atome ionisé, ou ,encore d'un champ localisé mais intense 'atome

(1) Ceci suppose le domaine $D$ fini. Lorsque $D$ devient infini, la zône de validité de la loi asymptotique est envahie par le spectre continu, où le numérotage des valeurs propres devient impossible. neutre éloigné), la eritique ilu paragraphe 2 s'applique et il faut tenir compte de l'influence des valeurs spectrales fortement perturbées.

Peut-on corriger l'erreur en combinant les formules (7) et (9)? Tout dépend de la déformation subie par la courbe $W_{p}$, et des valeurs des éléments $\boldsymbol{H}^{1}{ }^{10}$. La formule (7) est valable pour les très pelites et les trìs grandes valeurs de $p$, les formules $(9)$ pour les valeurs de $p$ correspondant (') aux valeurs propres de petits indices du deuxième champ, mais il y a des valeurs intermédiaires de l'indice $p$, que nous avons écartées jusqu'ici, qui donneront pour $W_{0}^{\prime}$ une correction qu'on ne pourra évaluer ni par (7) ni par (9). Il semble donc illusoire de chercher à calculer la correction du deuxième ordre par la méthode de $\mathbf{R}$. sauf si l'on a des raisons d'affirmer a priori que les termes ( 7 ) ou (9) compteront seuls.

Supposons que la perturbation soit constituée par un potentiel $V$ Le vecteur $V_{.0}$ devient, dans le langage de la mécanique ondulatoire. la fonction $V \Psi_{0}$, si $\Psi_{"}$ désigne la fonction propre étudiee. Il se pourra que $V \Psi^{*}$ soit, toute entière, localisée dans une région de l'espace où seront prépondérantes, soit les fonctions propres faiblement perturbées, soit les fonctions propres fortement perturbees. Dans le premier cas, (7) représentera pratiquement toute la correction (2), dans le deuxième cas se sera (9). Autrement on devra renoncer à la deuxième approximation.

b) Diffusion des électrons rapides par un champ coulombien $\left(^{3}\right)$. - La grosse difficulté, dans la théorie de la diffrastion des ondes de De Broglie, est de formuler les conditions aux limites : Les flux ont des expressions quadratiques, il n'est donc pas possible de se donner les flux incidents à travers une surface éloignée. On sera réduit à des hypothèses imparfaites. Nous allons poser le problème ainsi : Nous admettons qu'on peut représenter un pinceau électronique par une certaine fonction propre. d'énergie positive, définie par l'équation $د \Psi=W \Psi=K^{2} \Psi$ et un domaine $D$, très étendu. Nous chercherons comment elle sera per-

(1) Cette correspondance peut être établie, par exemple, en introduisant $H^{\prime}$ d'une manière continue. Mais elle ne joue pas de rôle, l'essentiel est. qu'on a la certitude de trouver, à partir d'un indice suffisamment élevé, les valeurs spectrales du deuxième problème

$\left.{ }^{2}\right)$ Ce raisonnement montre pourquoi les approximations supérieures des formules $R$ sont valables dans un problème tel que l'effet Stark, Zeeman. ou la dispersion : Les termes perturbateurs, petits au voisinage de l'atome, sont infinıment étendus et peuvent mème devevir infinis à l'infini. Les valeurs spectrales d'indices très élevées sont considérablement modifiées. Mais la variation de l'ex sonentielle l'emportera toujours de beaucoup sur la variation du potentiel perturbaleur. Et la fraction de la correction, qu'on ne peut estimer, devient ainsi négligeable

(3) Voir : Gornon. Z Physik, 1928, 48, 180. - L. dв Brocure: Ann. Inst. Pouncaré, 19.33, 3, 349. J. Winten, Ann. Phystque, décembre 1934 et Journal de Physique, 1935, 16, p. 71. Nous chorsissons les unités de manière, que $8 \pi^{2} m / h^{2}$ soit un coefficient numérique que nous n'écrivons pas, et appeions $K$ l'impuision. 
turbée par l'introduction du potentiel $V$. Supposons que $D$ et $V$ ont la symétrie sphérique autour de 0 , et prenons des coordonnées polaires de centre 0 . Les fonctions propres primitives auront l'expression

$$
\varphi\left(n, \boldsymbol{K}_{p}, \boldsymbol{m}\right)=J_{n+\frac{1}{2}}\left(K_{p} r^{r}\right) P_{n}^{m}(\cos \Theta) \frac{e^{+i m=}}{\sqrt{K_{p} R}}
$$

.J et $P$ fonctions de Bessel et Legendre, $K_{p}^{2}$ seront les valeurs propres du domaine $D$, définies par

$$
J_{n+\frac{1}{2}}\left(K_{p} R\right)=0
$$

si $\boldsymbol{R}$ est le rayon de $\boldsymbol{D}$. Une onde sera d'autant plus perturbée qu'elle sera localisée plus près de 0 ; cela signifie. pour $K_{p}$ donné, $n$ plus petit. Les perturbations seront encore très faibles pour les très grandes valeurs de $\boldsymbol{K}_{\boldsymbol{p}}$. Un pinceau monocinétique sera représenté par une certaine série.

$$
\Psi^{0}=\underset{n, m}{\Sigma_{n, m}} c_{\text {巳 }}\left(n, K_{p}, m\right)
$$

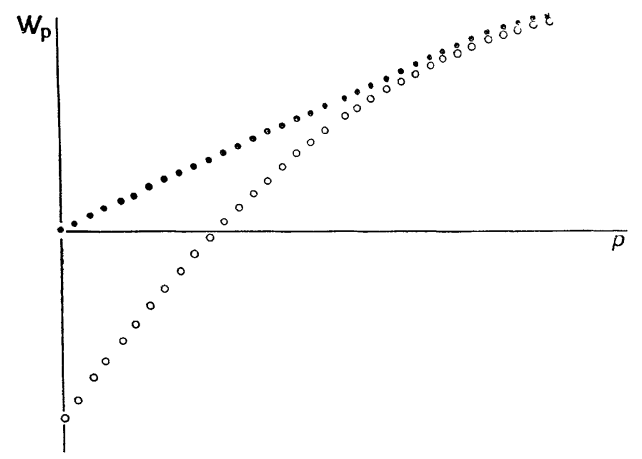

Fig. 2.

Si le champ $V$ est suffisamment intense pour perturber nolablement les ondes de petits indices (et c'est le cas dans le problème de la difiraction par un atome), nous pourrons appliquer les conclusions précédentes à la théorie des perturbations de R : En effet, on peut parlager les ondes en ondes très perturbées, qui correspondront notamment, aux petites valeurs le l'indice $n$, et en ondes peu perturbées, qui comprendront les grandes valeurs de $K_{p}$. Soit $\Psi_{0}$ une telle onde. Que peut-on dire de la correction sur $K_{p}$ ? Cette correction tendra vers zéro. 1 . Si $K_{b}$ tend vers l'infini; 2 . Pour $K_{p}$ donné, si le domaine $D$ s'étend indéfiniment; l'indice croît alors aussi indéfiniment. Si donc on étudie une onde d'énergie donnée, posilive, on peut toujours la négliger.

Que peut-on dire de la correction $\Psi^{\prime}$ et des deuxièmes approximations? Dans le développement de $V \Psi^{0}$, les? très perturbées (petites valeurs de $K_{p}$ et de $u$ ) ne peuvent être écartées a priori, en raison de la forme de $V$. Nous ne pourrons donc passer en seconde approximation. $\Psi^{1}$ nous échappera aussi en partie.
Mais nous voyons que les composantes que nous pouvons calculer, sont prédominantes aux grandes distances de $O$ (grands $n$ ). Ceci constitue une explication intuitive du fait suivant: On ne peut calculer que les valeurs d'une seule correction $\Psi^{1}$, et cela, seulement lorsque $K_{p}^{-2}$ est grand, comparé à $V$. Il nous reste maintenant, à comparer la méthode de $R$. et la méthode de Born. pour laquelle on a été concluit à un résultat analogue.

La figure 2 représente la courbe IIdans le cas actuel avec le choix d'axes ci-dessus.

4. Comparaison avec la méthode de Born. Nous avons traité ici le problème de la diffraction électroniqne, comme un problème spectral. Dans notre thèse, nous l'avons considéré, soit commeun problème de propagation d'ondes sphériques convergentes et divergentes, soit comme un problème de Dirichlet de caractère spécial. Le domaine devient infini, et la fonction de Green est déterminée d'une manière unique, grâce à la condition d' "Ausstrahlung " ('). Nous voulons montrer ici l'analogie qui existe entre le problême de Dirichlet et le problème spectral, pour justifier les raisonnements du paragraphe précédent.

Pour cela, nous partons du problème de Dirichlet. et considérons un domaine $D$ fimi. On part d'une fonction $\Psi^{0}$ vérifiant $\triangle \Psi^{\circ}=W^{\top} \Psi$, qui prend des valeurs imposées sur la surface extérieure $S$ de $D$, et correspondant à une valeur du paramètre $W$, qui n'est pas une valeur propre de $D\left({ }^{2}\right)$. On introduit le potentiel $V$ $(\Delta \Psi=(W-V, \Psi)$ et on cherche la modification subie par $\Psi^{0}$, les conditions aux limites restant inchangées. A la valeur $W$ correspond une fonction de Green $G$. bien déterminée, qu'on peut développer en série des fonctions propres de $\boldsymbol{D}$.

$$
G(P, Q)=\Sigma \Psi_{p}^{*} \frac{\Psi^{*}\left(() \Psi_{p}(P)\right.}{W-W_{P}}
$$

$p$ indice des valeurs propres. $P$ point courant, et $Q$ point source (voir Sommerfeld, loc. cit.). La méthode des approximations successives donne une formule de récurrence entre les approximations $\Psi^{n}$ et $\Psi^{n-1}$, qui s'écrit, si l'on y remplace la fonction de Green par son expression ci-dessus :

$$
\Psi^{n}(\boldsymbol{P})=\int_{D} \Psi^{n-1}(Q) V(Q) \sum_{p} \frac{\Psi_{p}^{*}(Q) \Psi_{p}^{*}(\boldsymbol{P})}{W-W_{p}^{-}} d v_{(}
$$

et si la série $\Sigma \Psi^{n}$ converge absolument, elle donnera la fonction vérifiant l'équation d'onde perturbée et prenant sur $S$ les valeurs imposées à $\Psi^{\prime 0}$. (11) peut s'écrire

$\Psi^{n}(\boldsymbol{P})=\sum_{p} \frac{\Psi_{p}(\boldsymbol{P})}{W-\boldsymbol{V}_{p}} \int_{D} \Psi^{n-1}(?) V(()) \Psi_{p}^{*}(()) d v_{p}(1 \geq)$

(1) Sommenect. Jahresberichte d. Deut. Wath. Veremi,unq, 1912. (2) Les fonctions propres sont les solutions de $\Delta \Psi=H^{\circ} \Psi, H^{r}$ quelconque, et s'antulant sur $S$ 
Considérons maintenant $\Psi^{n}$ comme un vecteur dans l'espace fonctionnel défini par les fonctions propres $\Psi_{p}$ de $D$ et écrivons l'expression de la composante d'indice $p$ de ce vecteur, qui se déduit facilement de l'équation ci-dessus,

$$
\Psi_{p}^{n}=\frac{1}{W-W_{p}}\left[V \Psi^{n-1}\right]_{p}
$$

(En adoptant la mème notation vectorielle pour l'intégrale du $2^{e}$ membre, qui n'est autre que la $p^{e} \mathrm{com}$ posante du vecteur $V \Psi^{n n-1}$.) Cette expression est analogue au dernier terme de la $2^{\mathrm{e}}$ équation (1). Mais $1^{\circ}$ ici $I^{\top}$ ne peut pas être une valeur propre, ni $\Psi^{0}$ une fonction propre; $2^{\circ}$ les termes en $W^{l}$ sont supprimés.

Bien que les problèmes posés soient €omplètement différents, il y a analogie formelle des premières approximations. (Les $W^{\imath}$ n'apparaissent qu'au second ordre). Supposons que $\Psi^{0}$ et $W$ soient très voisins $\left(^{1}\right)$

(1) $\Psi^{\circ}$ doit prentre des valeurs différentes de zéro sur la surface extérieure $S$ de $D$, alors que, par définition, dans le problème spectral. $\Psi_{\text {, }}$ s y annule. On peut concevoir $\psi^{\circ}$ comme provenant de la déformation subie par $\Psi_{j}$, si on augmente légèrement le domaine $D$. Ces raisonnements et définitions ne sont pas rigoureux. de la fonction propre $\Psi_{j}$ du domaine $\mathrm{D}$, de valeur propre $W_{i}$. Les composantes de $V \Psi^{0}$ et $V \Psi_{j}$ seront alors pratiquement les mèmes et on aura mèmes composantes $\Psi^{{ }^{1}}{ }_{p_{j}}$ (spectral) et $\Psi^{1}{ }_{p}$ (Dirichlet), pourva que

$$
W_{p}-\mathrm{H}^{\circ}>\mathrm{W}-\mathrm{H}_{j}{ }_{j}
$$

Ainsi, seules les valeurs propres voisines de $W$ donneront des composantes différentes. Sont elles importantes, dans le développement de $V$ Y'o? Cela dépend de la forme de la fonction $V$. Si $V$ est une fonction régulière et lentement variable, elles seront prépondérantes. Mais, dans un problème où $V$ varie très rapidement (Ici, $V$ a un pôle), il n'en sera plus de même et les deux méthodes pourront donner pour $\Psi^{\mathbf{1}}$ des résultats équivalents.

Nous examinerons, ailleurs, l'allure des phénomènes pour un domaine infini.

Nous remercions vivement M. L. de Broglie avec qui nous avons eu d'intéressantes et utiles discussions. 